\title{
Skilled Intuition and Selling Performance: An Abstract
}

\author{
Zachary R. Hall, Harish Sujan, and Michael Ahearne
}

\begin{abstract}
Approximately one in every nine jobs in the United States involves selling. As a result, significant research has focused on identifying determinants of salesperson performance ranging from adaptive selling, customer orientation, emotional intelligence, motivation, to job fit. Our research introduces and tests a new determinant of salesperson performance, skilled intuition. Drawing from naturalistic decision-making research, we define skilled intuition as a heightened ability to make accurate, intuitive judgments based on the pattern-recognition-matching process of environmental cues (i.e., customer cues) to schemas.

We investigate three intuitive judgments made by salespeople of their customers: intuition of needs, budget, and purchase likelihood. This paper reviews prior research on NDM research to suggest that top performing salespeople possess skilled intuition. In doing so, we highlight the importance of skilled intuition to both theory and practice, integrate prior research on determinants of salesperson performance, and provide managers with guidance on how to cultivate skilled intuition in their sales force.
\end{abstract}

\footnotetext{
Z.R. Hall $(\bowtie)$

Texas Christian University, Fort Worth, TX, USA

e-mail: z.hall@tcu.edu

H. Sujan

Tulane University, New Orleans, LA, USA

e-mail: hsujan@tulane.edu

M. Ahearne

University of Houston, Houston, TX, USA

e-mail:mahearne@uh.edu 